\title{
Monitoreo continuo de glucosa de seis días en pacientes diabéticos tipo 2 bajo hemodiálisis en tratamiento con insulinas en el Hospital de San José. (Bogotá)
}

\author{
Continuous glucose monitoring over a 6-day period in insulin-treated \\ patients with type 2 diabetes on haemodialysis at San José Hospital, Bogotá
}

\author{
Andrés Jaramillo Nieto ${ }^{1}$; Adriana Medina Orjuela ${ }^{2}$; Carlos Rosselli San Martin ${ }^{3}$; William Rojas \\ García ; Carlos David Centeno García ${ }^{5}$ Luisa María Montoya Quesada ${ }^{6}$
}

${ }^{1}$ Instructor Asociado Servicio de Endocrinología. Fundación
Universitaria de Ciencias de la Salud FUCS - Hospital de San José.
${ }^{2}$ Instructor Asociado Servicio de Endocrinología. Fundación
Universitaria de Ciencias de la Salud FUCS - Hospital de San José.
${ }^{3}$ Instructor Asociado Servicio de Nefrología. Fundación
Universitaria de Ciencias de la Salud FUCS - Hospital de San José.
${ }^{4}$ Profesor Asociado Servicio de Endocrinología. Fundación
Universitaria de Ciencias de la Salud FUCS - Hospital de San José.
${ }^{5}$ Residente de Nefrología. Fundación Universitaria de Ciencias de la
Salud FUCS - Hospital de San José
${ }^{6}$ Instructor Asistente División de Investigaciones. Fundación
Universitaria de Ciencias de la Salud FUCS - Hospital de San José.

Autor de correspondencia: Andres Jaramillo Nieto. Email: mdjaramillo5@yahoo.com

Fecha de recepción: 12/08/2018

Fecha de aceptación: 9/09/2018

\section{Resumen}

Objetivo: Determinar el comportamiento de la glucosa mediante monitoreo continuo de glucosa (MCG) en pacientes diabéticos tipo 2 en hemodiálisis (HD) bajo tratamiento con insulinas en el Hospital de San José - Unidad Renal Fresenius Medical Care.

Materiales y métodos: Estudio descriptivo longitudinal. Se incluyeron, desde abril hasta agosto de 2016, pacientes con diabetes tipo 2 con falla renal en HD tratados con insulinas. Se midieron como variables de interés: hipoglucemia grave, severa o asintomática, hiperglucemia, variabilidad glucémica, características demográficas y paraclínicas.

Resultados: Se incluyeron 25 pacientes, el 72\% fueron hombres, los niveles de hemoglobina glucosilada (HbA1c) fueron 8,35\% $( \pm 2,34)$. Con el MCG se apreció alta variabilidad, tendencia a la hiperglucemia en $67,7 \%$ de los datos y descenso durante la HD de la glucemia tisular. Se detectaron 52 episodios de hipoglucemia, los cuales fueron más frecuentes en los días sin diálisis y en horario diurno. Hubo una menor relación de episodios de hipoglucemia con el uso de insulina glargina

Conclusiones: El MCG en pacientes diabéticos en HD detectó alta variabilidad glucémica, hiperglucemia que predominó en los días de no diálisis y mayor número de hipoglucemias comparándola con la toma de glucometrías.

Palabras clave: Diabetes mellitus, insuficiencia renal crónica, diálisis, monitoreo, glucosa.

\section{Abstract}

Objective: To determine glucose behaviour using continuous glucose monitoring (CGM) in insulin-treated patients with type 2 diabetes on haemodialysis (HD) at San José Hospital - Fresenius Medical Care Renal Unit.

Materials and methods: Longitudinal descriptive study conducted between April and August 2016, including insulintreated patients with type 2 diabetes and renal failure on haemodialysis. The variables measured included severe or asymptomatic hypoglicaemia, hyperglicaemia, blood sugar variability, demographic characteristics and paraclinical variables.

Results: Overall, 25 patients were included and 72\% of them were males. Glycosylated haemoglobin (HbA1c) levels were $8.35 \%( \pm 2,34)$. CGM showed high variability, with the data showing a $67.7 \%$ trend towards hyperglicaemia, and alowering of tissue glucose during HD. There were 52 hypoglycaemic episodes detected, with a higher frequency during the days without dialysis and during the day. There was a lower ratio of hypoglicaemic episodes with the use of insulin Glargine.

Conclusions: CGM in diabetic patients on HD detected high blood sugar variability, hyperglycaemia predominantly during non-dialysis days, and a higher number of hypoglycaemic episodes when compared with blood glucose measurements.

Key words: Diabetes mellitus, chronic renal failure, dialysis, monitoring, glucose. 


\section{Introducción}

Actualmente, la diabetes mellitus tipo 2 (DM2) se ha convertido a nivel mundial en una pandemia, pasando de $246 \mathrm{mi}-$ llones de pacientes en el año 2006 a cerca de 415 millones en el año $2015^{(1)}$, no siendo Colombia ajena a esta situación con cerca de 1,9 por 100 habitantes en el año 2015, con una prevalencia del $9,6 \%$, lo cual ocasiona un impacto negativo en la sociedad con más de 19.802 muertes relacionadas y con un costo de 1200 dólares al año por paciente ${ }^{(2)}$.

En el mundo, la diabetes es la principal causa de enfermedad renal crónica, falla renal terminal y requerimiento de terapia de remplazo renal y el número de casos está incrementándose rápidamente. De los nuevos casos de enfermedad renal terminal anualmente en Estados Unidos (EE.UU.), cerca del $44 \%$ es debido a la diabetes ${ }^{(3)}$. En Colombia, según los datos de la Cuenta de Alto Costo del 2015, la prevalencia de falla renal terminal es de 61,62 por 100.000 personas, con un aumento en los últimos años. El número de pacientes diagnosticados con insuficiencia renal estadio 1 al 5 fue de 770.428, de los cuales el 28,6\% eran diabéticos; de los pacientes en falla renal estadio 5 , el $24,2 \%$ eran diabéticos ${ }^{(2)}$.

El paciente en falla renal en hemodiálisis (HD) requiere un manejo multidisciplinario que incluye control de las cifras tensionales, manejo de comorbilidades y un adecuado control metabólico, el cual ha demostrado disminuir la mortalidad en este grupo de pacientes ${ }^{(4,5)}$; sin embargo, tanto el mal control metabólico como la búsqueda de mejores metas de hemoglobina glucosilada (HbA1c) que aumentan el riesgo de hipoglucemias, pueden generar impacto en la mortalidad(6), por lo tanto, las terapias utilizadas deben brindar el mejor margen de seguridad y una menor tasa de hipoglucemias ${ }^{(7)}$.

La monitorización actual de los pacientes diabéticos en HD está basada en la automedición de la glucosa sanguínea capilar que demuestra el estado actual de la glucemia sin brindar la posibilidad de determinar variaciones durante el procedimiento o presencia de hipoglucemias asintomáticas y los niveles de $\mathrm{HbA1c}$ que pueden ser alterados debido a los requerimientos transfusionales y utilización de eritropoyetina, entre otros. Por todo lo anterior, el monitoreo continuo de glucosa (MCG) ha sido reportado como un método útil para monitorizar los niveles de glucosa en pacientes en HD o diálisis peritoneal y este determina la variabilidad glucémica y la incidencia de los episodios de hipoglucemia antes, durante y después de las sesiones de $\operatorname{HD}^{(8,9)}$.

Existen muy pocos estudios realizados en pacientes diabéticos en HD que incluyan $\mathrm{MCG}^{(10-13)}$, estos han logrado demostrar episodios de hipoglucemia durante la sesión de HD y posteriormente; sin embargo, todos han sido realizados en poblaciones europeas y norteamericanas. Se desconoce el comportamiento glucémico, incidencia de hipoglucemias (sintomáticas y asintomáticas) e hiperglucemias en pacientes lati- noamericanos con DM2 bajo tratamiento con insulina, antes, durante y después de las sesiones de HD.

Por lo tanto, se planteó la realización de un estudio descriptivo longitudinal en un hospital de cuarto nivel de Bogotá, incluyendo pacientes diabéticos tipo 2 con falla renal terminal en HD bajo tratamiento con insulina, a los cuales se les implantó un monitor continuo de glucosa tisular, con el objetivo de determinar el comportamiento de la glucosa antes, durante y después de las sesiones de HD, además de la detección de episodios de hipoglucemia asintomáticos, sintomáticos o severos e hiperglucemias.

\section{Materiales y métodos}

Estudio descriptivo longitudinal, realizado en la Unidad Renal (Fresenius Medical Care) del Hospital de San José, Bogotá.

Se incluyeron pacientes mayores de 18 años participantes en el programa de HD con duración mayor de seis meses, diagnóstico de DM2 mayor de seis meses y en tratamiento con insulina. Se excluyeron pacientes con patologías comórbidas descompensadas o procesos infecciosos intercurrentes activos (falla cardiaca, falla hepática, neumonía, infecciones urinarias, etc.), pacientes recientemente hospitalizados por complicaciones crónicas o agudas de la diabetes, incluyendo cetoacidosis, estado hiperosmolar o hipoglucemias severas en los últimos dos meses, utilización de glucocorticoides, diagnóstico de neoplasia (excepto cáncer de piel no melanoma) y pacientes con alteraciones cognitivas, inadecuada adherencia o entendimiento para la utilización del MCG o uso del glucómetro, inadecuado registro de la alimentación y ejercicio en el diario respectivo o contraindicación para implante de sensor de glucosa tisular.

Se definió un tamaño de muestra por conveniencia de 25 pacientes. Se seleccionaron los pacientes mediante muestreo aleatorio simple con remplazo y se les indicaron los objetivos del estudio, los beneficios y riesgos. Los pacientes que aceptaron participar en el estudio, firmaron el consentimiento informado y se programó una cita para realizar entrenamiento e implante del sensor de MCG de seis días, diligenciamiento del formato de recolección de datos, toma de variables antropométricas, revisión de la historia clínica electrónica del paciente y exámenes de laboratorio realizados en los últimos cuatro meses en el laboratorio de referencia utilizado por la unidad renal.

Se definieron las variables clínicas de hipoglucemia sintomática como paciente con síntomas clásicos de hipoglucemia acompañados de glucometría inferior o igual a $70 \mathrm{mg} / \mathrm{dl}$. Hipoglucemia asintomática se definió en un paciente asintomático con glucometría inferior o igual a $70 \mathrm{mg} / \mathrm{dl}$. La hipoglucemia severa se definió como cualquier episodio de descenso severo de glucosa en el cual el paciente requiriera asistencia de otra persona para administración de carbohidratos, glucagón u otras acciones. Hiperglucemia se definió como una glucemia mayor de lo esperado para el momento del día, mayor 
de $140 \mathrm{mg} / \mathrm{dl}$ preprandial y mayor de $180 \mathrm{mg} / \mathrm{dl} 2$ horas postprandiales $^{(14)}$. Se definió hipoglucemia medida por el sensor, como niveles de glucosa tisular menor de $70 \mathrm{mg} / \mathrm{dl}$ con una duración mayor de 20 minutos $^{(10)}$ y se definió alta variabilidad de la glucemia como una desviación estándar mayor de $28^{(15)}$. Todos los antecedentes y complicaciones de los pacientes se tomaron de la historia clínica.

Durante la fase de utilización del sensor los pacientes tomaron glucometrías para la realización de las calibraciones respectivas del mismo y el día fue dividido en periodos así: madrugada, desde las 00:01 hasta las 06:00 horas; periodo diurno, de 06:01 a 20:00 horas, y periodo nocturno, de 20:01 a 00:00 para la clasificación de las hipoglucemias por sensor y por toma de glucometrías.

\section{MCG y registro diario de actividades}

Todos los pacientes y cuidadores fueron entrenados en el funcionamiento básico del sensor, educación con respecto a los diagnósticos de hipoglucemia sintomática, asintomática, severa e hiperglucemia. Se les explicó la importancia de mantener siempre colocado el sensor durante todo el estudio (6 días), además del adecuado registro de las comidas, las glucometrías (que debían ser mínimo tres al día), actividad física y, cualquier tipo de sintomatología de hipoglucemia, en el diario de actividades.

A los pacientes que ingresaron se les implantó el sensor de MCG (Medtronic ENLITE Glucose Sensor Ref: MMT-7008) y se conectó al transmisor iPro2 Ref: MMT-7741, números de series: GT3070657M, GT307707718M o GT3027907M. (Medtronic, Minneapolis, MN, USA). Este procedimiento fue realizado en el servicio de procedimientos de sala de HD por parte de una enfermera especializada en entrenamiento de pacientes diabéticos y certificada en manejo de sensores de glucosa tisular.

Al completar los seis días de registro, a los pacientes se les retiró el dispositivo iPro2 y luego de su limpieza según las recomendaciones del fabricante (Medtronic, Minneapolis, MN, EE.UU.) se conectó a la estación de descarga de datos y por vía USB al computador con un programa informático (CareLink iPro medtronic $^{\mathrm{MR}}$ ), se obtuvieron los niveles de glucosa tisular registrada cada 5 minutos en gráficas, tablas de datos, curvas, desviaciones estándar y área bajo la curva mayor de $140 \mathrm{mg} / \mathrm{dl}$ y menor $70 \mathrm{mg} / \mathrm{dl}$, las cuales se exportaron a una tabla de Excel ${ }^{\circledR}$ para el análisis.

\section{Descripción de la hemodiálisis}

La prescripción de HD no difirió durante el estudio. Fueron usados filtros de alta eficiencia a base de membranas de Helixone, máquinas de diálisis de tipo 4008b con sistemas de conductividad tipo Online Clearance Monitoring $\left(\text { OCM }^{\circledR}\right)^{(16)}$, con parámetros de diálisis dados por flujos de bomba de la máquina $\mathrm{Qb} \geq \mathrm{a} 300 \mathrm{ml} / \mathrm{min}$ y flujos de líquido de dializado $\mathrm{Qd} \geq \mathrm{a}$
$500 \mathrm{ml} / \mathrm{min}$ a base de concentrados ácido estándar SLG 100 y bicarbonato estándar Bibag 4008, concentrado de glucosa en el dializado de 1,47 g/L, tiempo dialítico de 4 horas y temperaturas promedio de 36 grados. Para los cuatro pacientes de hemodiafiltración se utilizaron máquinas de online 5008 con uso de líquidos predilucionales de reposición para generación de fenómenos convectivos; máxima tasa de sustitución de 350 $\mathrm{ml} / \mathrm{min}$ y similares tiempos de sesión temperatura y flujos de bomba descritos anteriormente ${ }^{(17)}$.

\section{Análisis estadístico}

Se realizó un análisis descriptivo de la información. Las variables cuantitativas fueron reportadas como promedio y desviación estándar o como mediana y rango intercuartílico de acuerdo con la distribución de los datos; las variables cualitativas fueron reportadas por medio de frecuencias absolutas y relativas. Se elaboraron gráficas que describieron el comportamiento de los niveles de glucosa tisular.

Se reportaron las proporciones de los eventos de hipoglucemia e hiperglucemia durante y por fuera de la HD. Por otro lado, se analizó la frecuencia de los episodios de hipoglucemias por segmentos horarios (madrugada, 00:01 a 06:00, día; 06:01 a 20:00 y noche, 20:01 a 00:00).

Se estructuró una base de datos en Excel $^{\circledR}$ exportada al software estadístico STATA 13 para su análisis.

\section{Consideraciones éticas}

El estudio recibió la aprobación del Comité de Ética en Investigación con Seres Humanos del Hospital de San José (Bogotá) en el acta 22 del 2 de diciembre de 2015, además de la aprobación de la Dirección Médica Nacional de Fresenius Medical Care.

\section{Resultados}

De los 30 pacientes invitados a participar, 25 firmaron el consentimiento informado e ingresaron al estudio entre abril y octubre de 2016 . El $72 \%$ de los pacientes eran hombres; edad promedio de 61,5 años ( \pm 13.6$)$. Tenían diagnóstico de DM2 el 92\% y dos pacientes tenían diagnóstico de posible diabetes tipo LADA. La mediana del tiempo de diagnóstico confirmado de diabetes fue de 16 años (RIC: 10-23), el inicio de tratamiento con insulina de 10 años (RIC: 4-15) y el tiempo de inicio de la terapia dialítica de 4 años (RIC: 2-6) (tabla 1).

Dentro de las variables antropométricas, el promedio del índice de masa corporal (IMC) fue de $25,4 \mathrm{~kg} / \mathrm{m}^{2}( \pm 4,9)$. El $52 \%$ de los pacientes realizaban algún tipo de actividad física, con un tiempo promedio descrito por interrogatorio clínico de 38,3 minutos $( \pm 8,3)$ al día, las principales actividades físicas realizadas fueron caminata (28\%) y bicicleta estática (12\%).

El control de la glucemia reflejado en el examen de HbA1c presentó un promedio de $8,35 \%( \pm 2,34)$ (tabla 1$)$. 
Tabla 1. Características de los pacientes con diabetes tipo 2 en HD bajo tratamiento con insulinas

\begin{tabular}{|c|c|}
\hline Variable & N: $25(\%)$ \\
\hline Edad - años : promedio $\pm \mathrm{DE}$ & $61,5 \pm 13,6$ \\
\hline Sexo (Hombres) & $18(72 \%)$ \\
\hline \multicolumn{2}{|l|}{ Actividad económica } \\
\hline Pensionado - cesante & $18(72 \%)$ \\
\hline Amas de casa & $3(12 \%)$ \\
\hline Independiente & $2(8 \%)$ \\
\hline Comerciante & $1(4 \%)$ \\
\hline Oficios varios & $1(4 \%)$ \\
\hline $\begin{array}{l}\text { Tiempo diagnóstico DM - años: } \\
\text { Mediana (RIC) }\end{array}$ & $16(10-23)$ \\
\hline $\begin{array}{l}\text { Inicio de tratamiento con insulina- } \\
\text { años: Mediana (RIC) }\end{array}$ & $10(4-15)$ \\
\hline $\begin{array}{l}\text { Tiempo de inicio de la terapia dialítica- } \\
\text { años: Mediana (RIC) }\end{array}$ & $4(2-6)$ \\
\hline \multicolumn{2}{|l|}{ Complicaciones por diabetes } \\
\hline Nefropatía & $\begin{array}{c}\text { Todos los } \\
\text { pacientes del } \\
\text { estudio la tenían }\end{array}$ \\
\hline Neuropatía* & $17(68 \%)$ \\
\hline Retinopatía** & $12(48 \%)$ \\
\hline Enfermedad coronaria *** & $8(32 \%)$ \\
\hline Enfermedad cerebrovascular **** & $3(12 \%)$ \\
\hline HbA1c \% Promedio DE & $8,35 \pm 2,34$ \\
\hline Mayor a $8 \%$ & $12(48 \%)$ \\
\hline Entre $8 \%$ y $7 \%$ & $6(24 \%)$ \\
\hline Menor al 7\% & $7(28 \%)$ \\
\hline Colesterol total mg/dl: Mediana (RIC) & $150(127-188)$ \\
\hline (HDL) mg/dl: Mediana (RIC) & $36,6(27,3-40-3)$ \\
\hline LDL mg/dl: Mediana (RIC) & $78,56(61,2-115,2)$ \\
\hline Triglicéridos mg/dl: Mediana (RIC) & $181,1(125,11-213)$ \\
\hline Hemoglobina $\mathrm{g} / \mathrm{dl}$ - promedio $\pm \mathrm{DE}$ & $11,4 \pm 2,4$ \\
\hline Hematocrito $\%$ - promedio $\pm \mathrm{DE}$ & $34,2 \pm 6,9$ \\
\hline Creatinina $\mathrm{mg} / \mathrm{dl}$ - promedio $\pm \mathrm{DE}$ & $7,6 \pm 2,7$ \\
\hline BUN mg/dl - promedio $\pm \mathrm{DE}$ & $57,4 \pm 16,8$ \\
\hline
\end{tabular}

*Por síntomas de disestesias, parestesias o toma de medicación para neuropatía diabética, referido por paciente en entrevista médica o en historia clínica.

** Retinopatía diabética tratada con terapia de fotocoagulación, referido por el paciente en entrevista médica o en historia clínica.

***Antecedentes de angina, infarto agudo de miocardio o revascularización miocárdica. ****Antecedentes de evento cerebrovascular trombótico con déficit neurológico. DE, desviación, estándar; RIC, rango intercuartílico; BUN, nitrógeno ureico en sangre; DM, diabetes mellitus; HDL, colesterol de alta densidad; LDL, colesterol de baja densidad.
El tratamiento con insulina en los pacientes se distribuyó así: el 52\% de los pacientes utilizaban esquema basal, el 44\% basal bolo y solo un paciente basal plus. Las insulinas basales utilizadas fueron: Insulina Glargina en el 48\%, insulina NPH (Protamina Neutra de Hagedorn) en el 44\% e insulina Detemir y Degludec cada una en un paciente. Las insulinas rápidas utilizadas fueron: insulina Lispro en 5 pacientes, insulina Aspart en 3 pacientes, insulina Glulisina en 2 pacientes e insulina Cristalina en 2 pacientes (tabla 2).

Se analizaron 152 días de monitoría con sensor de los cuales 53 corresponden a los días en HD. El 37,7\% de las sesiones de HD fueron realizadas en el horario de la mañana, el 35,8\% en el horario de medio día y el $26,5 \%$ restante en el horario de la tarde. La duración promedio fue de 233 minutos $( \pm 18,3)$. No se presentaron reacciones alérgicas al sensor ni se requirió retiro anticipado de este por eventos adversos u hospitalizaciones.

Tabla 2. Insulinas utilizadas en los pacientes con diabetes tipo 2 en HD bajo tratamiento con insulinas

\begin{tabular}{|c|c|c|}
\hline Variable & $\mathbf{N}(\%)$ & Dosis UI (RIC) \\
\hline Esquema basal & $13(52 \%)$ & NA \\
\hline Basal bolo & $11(44 \%)$ & NA \\
\hline Basal plus & $1(4 \%)$ & NA \\
\hline \multicolumn{3}{|c|}{ Insulinas basales usadas } \\
\hline Glargina & $12(48 \%)$ & $17.5(13.8-20)$ \\
\hline NPH & $11(44 \%)$ & $15(10-26)$ \\
\hline Detemir & $1(4 \%)$ & 4 \\
\hline Degludec & $1(4 \%)$ & 25 \\
\hline \multicolumn{3}{|c|}{ Insulinas rápidas usadas } \\
\hline Lispro & $5(20 \%)$ & $24(9-30)$ \\
\hline Aspart & $3(12 \%)$ & $9(6-19,5)$ \\
\hline Glulisina & $2(8 \%)$ & $16,5(12,7-20,2)$ \\
\hline Cristalina & $2(8 \%)$ & $30,5(25,7-32,2)$ \\
\hline \multicolumn{3}{|c|}{ Insulinas usadas en esquema basal } \\
\hline $\mathrm{NPH}$ & $9(36 \%)$ & NA \\
\hline Glargina & $3(12 \%)$ & NA \\
\hline Degludec & $1(4 \%)$ & NA \\
\hline \multicolumn{3}{|c|}{ Insulinas usadas en esquema basal bolo } \\
\hline Glargina - Lispro & $5(20 \%)$ & NA \\
\hline Glargina - Aspart & $2(8 \%)$ & NA \\
\hline Glargina - Glulisina & $2(8 \%)$ & NA \\
\hline $\mathrm{NPH}$ - Cristalina & $2(8 \%)$ & NA \\
\hline \multicolumn{3}{|c|}{ Insulinas usadas en esquema basal plus } \\
\hline Glargina - Glulisina & $1(4 \%)$ & NA \\
\hline
\end{tabular}

UI, Unidades internacionales; RIC, Rango intercuartílico; NPH, protamina neutra de Hagedorn; NA, no aplica 
Tabla 3. Episodios de hipoglucemias por glucometrías

\begin{tabular}{|c|c|c|c|c|c|}
\hline \multicolumn{6}{|c|}{ Episodios de hipoglucemias por glucometrías } \\
\hline Hora & $\begin{array}{c}\text { 00:01 a 06:00 } \\
\text { Madrugada }\end{array}$ & $\begin{array}{c}\text { 06:01-20:00 } \\
\text { Diurno }\end{array}$ & $\begin{array}{c}\text { 20:01 -00:00 } \\
\text { Nocturno }\end{array}$ & Intradiálisis & Total \\
\hline Día diálisis & 1 & 4 & 0 & 0 & 5 \\
\hline Día no diálisis & 1 & 5 & 0 & 0 & 6 \\
\hline Total & 2 & 9 & 0 & 0 & 11 \\
\hline
\end{tabular}

\section{Datos de las glucometrías de los pacientes}

Se encontraron 514 glucometrías con un promedio de 20,5 glucometrías por paciente y se identificaron once episodios de hipoglucemias en siete pacientes. Se verificó el libro de registro de alimentos y ningún paciente presentó hipoglucemia severa que requiriera ayuda de otras personas, manejo farmacológico con glucagón o intrahospitalario. Dentro de las hipoglucemias reportadas, 9 de 11 fueron asintomáticas y no requirieron manejos adicionales; siete de las anteriores estuvieron presentes antes de la ingesta de alimentos y dos después de la cena.

Los episodios de hipoglucemia se presentaron con mayor frecuencia en los días de no diálisis (54,5\%). El momento del día en el cual se detectaron más episodios de hipoglucemia por glucómetro fueron: periodo diurno con 9/11 hipoglucemias y madrugada 2/11 hipoglucemias, no se presentaron episodios nocturnos (tabla 3 ).

Los esquemas de insulina utilizados en los pacientes que presentaron hipoglucemias fueron: basal bolo en el 63,6\% de los pacientes y basal en el 36,4\% restante.

\section{Datos del MCG}

Se excluyeron 22 días de 174 días disponibles, por haber presentado falla en la calibración y por pérdida de los datos del sensor, lo que impedía su análisis.

Se analizaron 40.485 datos de sensor. Se apreció una alta variabilidad de la glucosa tisular con una mediana $37,5 \mathrm{mg} / \mathrm{dl}$ (RIC: 27,2 - 55,75). En los días de HD, la mediana fue de $38 \mathrm{mg} / \mathrm{dl}$ (RIC: 31-58) y los días de no HD, la mediana fue de $37 \mathrm{mg} / \mathrm{dl}$ (RIC: $27-$ $54)$, con un promedio de glucosa tisular de $184 \mathrm{mg} / \mathrm{dl}( \pm 45,4)$.

El área bajo la curva por encima de $140 \mathrm{mg} / \mathrm{dl}$ presentó una mediana de 40,6 mg/dl (RIC: 16,8-75,8); los días de diálisis esta fue de 33,6 mg/dl (RIC: 13,8 - 53,5) y los días de no diálisis fue de 44,8 mg/dl (RIC: 18,1-85,5).

Con respecto a los valores de glucosa del sensor, el 1,85\% $(\mathrm{n}=747)$ se encontró por debajo de $70 \mathrm{mg} / \mathrm{dl}$, y el $67,8 \%$ $(\mathrm{n}=27430)$ por encima de $140 \mathrm{mg} / \mathrm{dl}$. Los días de HD, los valores del sensor se encontraron por debajo de $70 \mathrm{mg} / \mathrm{dl}$ en el 2,8 y por encima de $140 \mathrm{mg} / \mathrm{dl}$ en el 63,1\%. En los días de no HD, los valores del sensor se encontraron menores de $70 \mathrm{mg} / \mathrm{dL}$ en el 1,9\% y mayores de $140 \mathrm{mg} / \mathrm{dl}$ en el 68,8\%.
Al graficar los datos del sensor de todos los pacientes en una curva de 24 horas, se aprecia una tendencia a la hiperglucemia durante todo el día con un descenso de los niveles de glucosa entre las 4 a las 10 a.m. (figura 1A).

Analizando específicamente las horas de realización de terapia dialítica, se revisaron 2.532 valores de glucosa tisular del sensor, de estos valores, el 1\% se encontró con niveles menores de $70 \mathrm{mg} / \mathrm{dl}$, el $47 \%$ entre 70 y $140 \mathrm{mg} / \mathrm{dl}$ y el $52 \%$ mayores de $140 \mathrm{mg} / \mathrm{dl}$; adicionalmente valores mayores de $180 \mathrm{mg} / \mathrm{dl}$ se presentaron en el 21,1\%, apreciándose una tendencia a la hiperglucemia durante las sesiones de HD. Además, se apreció un descenso de la glucosa tisular con el paso de las horas en diálisis (figura 1B), considerando que el líquido de diálisis contiene glucosa.

Se detectaron un total de 52 episodios de hipoglucemias por el MCG, lo cual contrasta con solo 11 episodios de hipoglucemias registrados por datos de glucometrías. Estos episodios de hipoglucemias se presentaron en 11 pacientes, detectando 6 pacientes adicionales a los reportados por glucometrías.

La duración de los episodios de hipoglucemia, en promedio, fue de 50 minutos (RIC: $30-87,5$ ). De los 52 episodios de hipoglucemias detectados por sensor solo uno fue sintomático y no se presentaron episodios de hipoglucemias severas.

$\mathrm{Al}$ analizar los datos de las hipoglucemias detectadas por el sensor, estas fueron más frecuentes en el día de no diálisis (59,6\%). El momento del día en el cual se detectaron más episodios de hipoglucemia con sensor fueron: periodo diurno $57,6 \%$, madrugada $32,6 \%$ y nocturno 9,6\% Durante el seguimiento solo se presentaron dos episodios de hipoglucemia por sensor durante el procedimiento dialítico (tabla 4).

Dentro de las hipoglucemias detectadas por sensor, los diferentes esquemas usados de insulinoterapia fueron así: insulina NPH con 24 episodios de hipoglucemia, Detemir/Aspart con 11 episodios, Glargina/Lispro 8 episodios, Glargina/ Glulisina 5 episodios, NPH/Cristalina 3 episodios y Glargina/ Aspart un episodio.

\section{Datos según HbA1c e hiperglucemia}

Al discriminar por niveles de $\mathrm{HbA} 1 \mathrm{c}$, dos pacientes presentaron niveles de $\mathrm{HbA1c}$ menores de 7\% con ocho episodios 
Figura 1. Gráficos de MCG A. Mediana de glucosa tisular (24 horas de sensor) B. Mediana de glucosa tisular en hemodiálisis
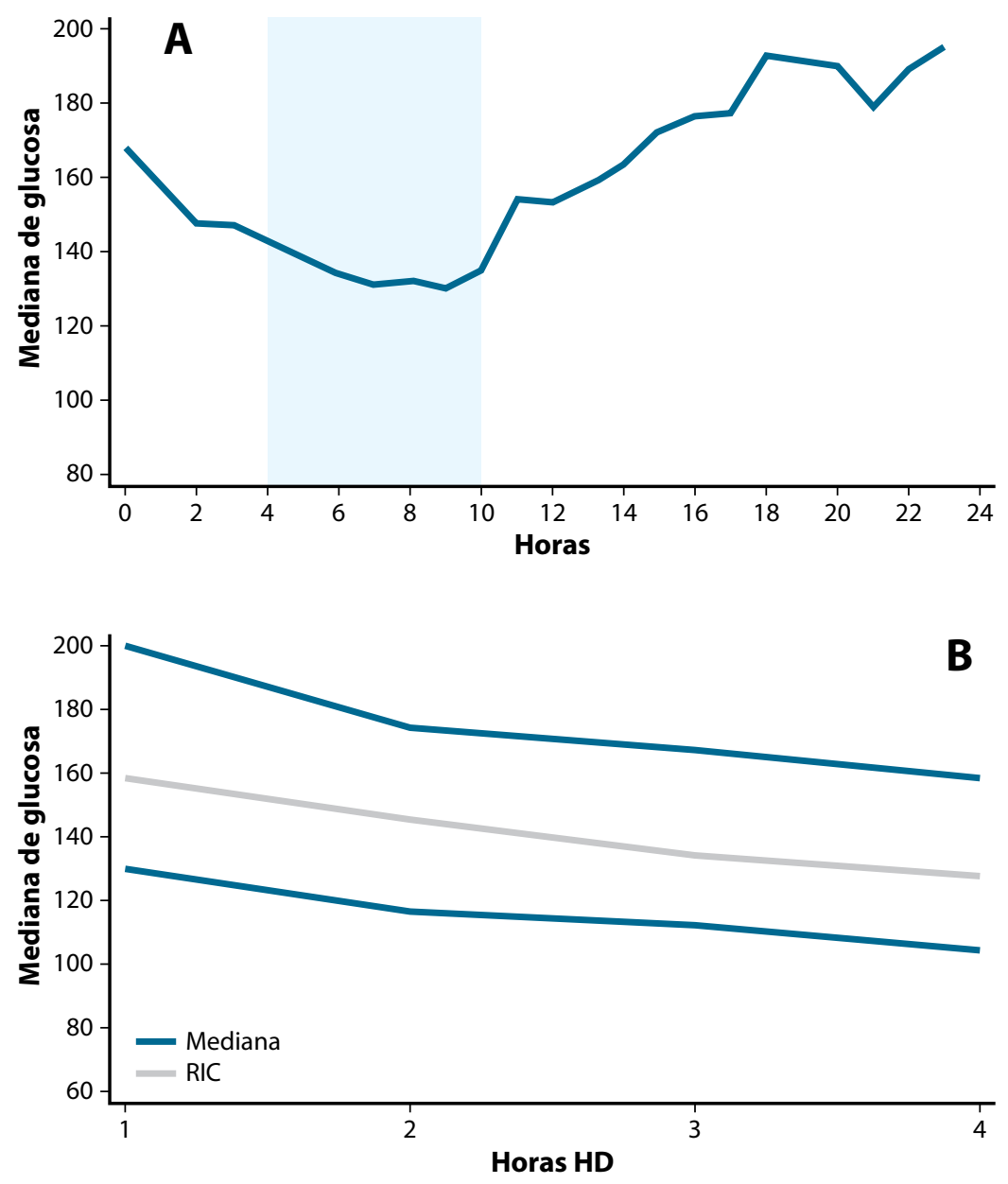

\section{Discusión}

En la actualidad, la diabetes se ha convertido en una pandemia global, con un número creciente de pacientes afectados y, como consecuencia, un aumento de las complicaciones secundarias $^{(1)}$. Dentro de estas, la nefropatía diabética tiene un alto impacto social debido al requerimiento de terapia dialítica, generadora de riesgo cardiovascular en los pacientes. El manejo de los pacientes en falla renal en HD requiere un enfoque multidisciplinario, incluyendo un adecuado control metabólico; sin embargo, uno de los factores limitantes más importantes es la hipoglucemia, por lo cual las terapéuticas utilizadas deben brindar el mejor margen de seguridad y una menor tasa de hipoglucemias ${ }^{(7)}$.

En la población evaluada, la mayoría de los pacientes no realizaban actividad laboral (72\%) o física (48\%), además tenían una dieta alta en carbohidratos, lo cual puede impactar de forma negativa para lograr la meta de HbA1c entre 7\% y 8\% y plantearía la posibilidad de realizar ajustes en actividad física y alimentación como una medida adicional a la farmacológica para llegar a un mejor control metabólico.

Observamos dentro de las variables paraclínicas un adecuado control de hemoglobina y hematocrito dentro de las metas para guías $\mathrm{KDIGO}^{(18)}$; (hemoglobina: 11 -13 g/ dl). El promedio visto en nuestros pacientes fue de 11,4 $\mathrm{g} / \mathrm{dl}( \pm 2,4)$, que se encuentra dentro de los

de hipoglucemia; dos pacientes presentaron niveles de HbA1c entre 7\% y $8 \%$ con 13 episodios de hipoglucemia y siete pacientes tenían niveles de HbA1c mayores de 8\% con 31 episodios de hipoglucemia.

La hiperglucemia fue predominante en el estudio. Del total de datos de glucosa tisular, el 67,7\% se encontró en valores de glucosa del sensor mayores de $140 \mathrm{mg} / \mathrm{dl}$, lo cual fue más frecuente en los días de no realización de HD $(68,8 \%)$, en comparación con los días de sesión dialítica con un 63,1\%. rangos aceptados para normalidad en paciente en HD. Este dato pudiese reflejar confiabilidad de los valores de HbA1c, que pueden, en casos de anemia, verse subvalorados en los pacientes renales crónicos; sin embargo, el realizar MCG en nuestros pacientes nos permitió demostrar una alta variabilidad glucémica con frecuentes episodios de hiperglucemia e hipoglucemia, lo cual lleva a que los valores reportados de HbA1c puedan estar alterados debido a esta fluctuación y no expresar el control metabólico real del paciente.

Tabla 4. Episodios de hipoglucemias por sensor tisular

\begin{tabular}{l|c|c|c|c|c}
\multicolumn{7}{c}{ Hora } & $\begin{array}{c}\text { E0:01 a 06:00 } \\
\text { Madrugada }\end{array}$ & $\begin{array}{c}\text { 06:01-20:00 } \\
\text { Diurno }\end{array}$ & $\begin{array}{c}\mathbf{2 0 : 0 1 - 0 0 : 0 0} \\
\text { Nocturno }\end{array}$ & Intradiálisis & Total \\
\hline Día diálisis & 10 & 6 & 3 & 2 & 0 \\
\hline Día no diálisis & 7 & 22 & 2 & 2 & 31 \\
\hline Total & 17 & 28 & 5 & 52 \\
\hline
\end{tabular}


El MCG nos permite apreciar cómo los valores de glucosa tisular presentan una tendencia al descenso durante este procedimiento, muy similar a estudios $\operatorname{previos}^{(8,12,13,19)}$, pero en contraste, no se aumentaron los episodios de hipoglucemia durante la sesión de HD o durante el día de diálisis, posiblemente por la tendencia a la hiperglucemia en los pacientes del estudio y al empleo de baños de diálisis con glucosa.

El MCG detectó un número mayor de episodios de hipoglucemias (52 contra 11 solo con glucometrías), lo cual es similar a lo reportado en la literatura ${ }^{(8,12)}$. Paradójicamente encontramos más episodios de hipoglucemias en los días de no diálisis, aunque la mediana de tiempo de duración de dichos episodios fue mayor en los días de HD (55 minutos), comparándolo con los días de no diálisis (45 minutos). Este postulado arroja interrogantes, ya que al mostrarse una tendencia hacia un mejor control glucémico en los días de diálisis, se podrían generar hipótesis alternas a este comportamiento. La existencia de agentes endógenos urémicos en altas concentraciones de tipo guanidina, monoguanida y biguanida, y la identificación de moléculas como el ácido 3 guanidinopropiónico; metabolito endógeno que estimula la secreción de insulina y genera sensibilización a la misma ${ }^{(20)}$, podrían explicar el fenómeno de hipoglucemias reflejadas en las horas previas a hemodiálisis.

Adicionalmente, el MCG le podría permitir al clínico realizar ajustes en el manejo para lograr un mejor control metabólico, evitando en lo posible las hiperglucemias sin incurrir en un mayor número de hipoglucemias.

Deberíamos plantear en los pacientes renales crónicos otras alternativas de terapia de remplazo renal que se realicen con más frecuencia, o mayor duración, como lo plantearon los estudios de Eloot y col., en el cual se mostraban mejores tasas de eliminación de metabolitos con el aumento de la duración de la diálisis para toxinas como la metilguanidina o el aumento de la frecuencia de las sesiones para mejorar la eliminación del ácido guanidinosuccínico ${ }^{(21)}$.

Es importante recalcar que dos pacientes que presentaron hipoglucemias con registro de glucometrías no fueron detectados con el monitoreo continuo y seis hipoglucemias por glucómetro no fueron detectadas por el monitoreo continuo. Esto puede deberse a que la mayoría de las hipoglucemias por glucometrías se encontraban cerca al valor de $70 \mathrm{mg} / \mathrm{dl}$, se presentaron antes de las comidas y el paciente consumió alimentos que evitaron continuar la pendiente de descenso que pudiese ser registrada por el sensor. Además, el registro de la glucosa tisular tiene un atraso de 10 minutos con respecto al registro de la glucosa capilar que pudo haber influido en el no registro del episodio por el sensor.

Llama la atención la utilización de insulinas humanas basales para el control metabólico NPH de los pacientes y el alto número de episodios de hipoglucemia en los pacientes que las utilizaban, por lo cual, cambiar a análogos de acción prolonga- da, podría disminuir los episodios de hipoglucemia debido a su mejor perfil de seguridad y farmacocinética.

Este es uno de los primeros estudios de esta naturaleza en Latinoamérica y demuestra un comportamiento muy similar de la glucosa tisular de los pacientes diabéticos en HD que los de otros países, pero nos diferenciamos en tener mayor número de episodios de hipoglucemias los días de no diálisis y una mayor tendencia a la hiperglucemias y variabilidad. Así mismo, este estudio presenta algunas limitaciones como el bajo tamaño de la muestra, la baja adherencia a la toma de glucometrías en algunos de los pacientes que limitó una mayor inclusión de días de monitoreo, debido a la necesidad que el sensor tiene para comparar sus datos con muestras de glucometrías tomadas por parte de los sujetos del estudio. Esta pérdida del sensor fue de 22 días, lo cual puede impactar en los resultados de nuestro estudio. En algunos de los pacientes se presentó, además, una baja adherencia a los tiempos de HD establecidos por las guías KDIGO (4 horas), variable que puede impactar en los desenlaces de hipoglucemia e hiperglucemia secundaria a un subóptimo aclaramiento de toxinas urémicas, además de no realizar intervenciones farmacológicas durante el monitoreo que han demostrado mejorar el control glucémico sin aumentar los episodios de hipoglucemia ${ }^{(13)}$.

\section{Conclusiones}

El MCG en pacientes diabéticos tipo 2 insulinorrequirientes en HD logró demostrar una tendencia a la hiperglucemia durante todo el día, descenso de los niveles de glucosa durante la diálisis y un mayor número de hipoglucemias que las registradas con el glucómetro, lo cual permitirá al clínico realizar ajustes en el manejo para lograr un mejor control metabólico, sin incurrir en un mayor número de hipoglucemias.

\section{Agradecimientos}

Agradecemos de forma especial a la señora Luz Amina Ely por toda su colaboración en la inserción de los sensores, recolección de datos y a Fresenius Medical Care por su colaboración con permitirnos acceder a sus bases de datos junto con la ayuda del personal de enfermería de las salas de HD.

\section{Declaración de conflicto de interés}

Los autores declaran no tener ningún tipo de conflicto de interés en este tema.

\section{Declaración de financiación del proyecto}

Este proyecto recibió financiación para la realización de traducción al idioma inglés, corrección de estilo y sometimiento del mismo por pares académicos, por parte de la Fundación Universitaria de Ciencia de la Salud FUCS, Bogotá Colombia. 


\section{Referencias}

1. Federación Internacional de la Diabetes. Atlas de la diabetes de la FID. Federación Internacional de la Diabetes; 2015. p. 144.

2. Fondo colombiano de enfermedades de alto costo. Situación de la enfermedad renal crónica en Colombia 2015. Colombia: Fondo colombiano de enfermedades de alto costo; 2015. p. 152

3. Sego S. Pathophysiology of diabetic nephropathy. Nephrol Nurs J. 2007;34(6):631-3.

4. Oomichi T, Emoto M, Tabata T, Morioka T, Tsujimoto Y, Tahara H, et al. Impact of glycemic control on survival of diabetic patients on chronic regular hemodialysis: a 7-year observational study. Diabetes Care. 2006;29(7):1496-500.

5. Ishimura E, Okuno S, Kono K, Fujino-Kato Y, Maeno Y, Kagitani S, et al. Glycemic control and survival of diabetic hemodialysis patients--importance of lower hemoglobin A1C levels. Diabetes Res Clin Pract. 2009;83(3):320-6.

6. Hill CJ, Maxwell AP, Cardwell CR, Freedman BI, Tonelli M, Emoto M, et al. Glycated hemoglobin and risk of death in diabetic patients treated with hemodialysis: a meta-analysis. Am J Kidney Dis. 2014;63(1):84-94.

7. Lim AKh. Diabetic nephropathy - complications and treatment. Int J Nephrol Renovasc Dis. 2014; 7:361-81.

8. Sobngwi E, Ashuntantang G, Ndounia E, Dehayem M, Azabji-Kenfack M, Kaze $\mathrm{F}$, et al. Continuous interstitial glucose monitoring in non-diabetic subjects with end-stage renal disease undergoing maintenance haemodialysis. Diabetes Res Clin Pract. 2010;90(1):22-5.

9. Skubala A, Zywiec J, Zełobowska K, Gumprecht J, Grzeszczak W. Continuous glucose monitoring system in 72-hour glucose profile assessment in patients with end-stage renal disease on maintenance continuous ambulatory peritoneal dialysis. Med Sci Monit. 2010;16(2):CR75-83.

10. Jung HS, Kim HI, Kim MJ, Yoon JW, Ahn HY, Cho YM, et al. Analysis of hemodialysis-associated hypoglycemia in patients with type 2 diabetes using a continuous glucose monitoring system. Diabetes Technol Ther 2010;12(10):801-7.

11. Kazempour-Ardebili S, Lecamwasam VL, Dassanyake T, Frankel AH, Tam FW,
Dornhorst A, et al. Assessing glycemic control in maintenance hemodialysis patients with type 2 diabetes. Diabetes Care. 2009;32(7):1137-42.

12. Gai M, Merlo I, Dellepiane S, Cantaluppi V, Leonardi G, Fop F, et al. Glycemic pattern in diabetic patients on hemodialysis: continuous glucose monitoring (CGM) analysis. Blood Purif. 2014;38(1):68-73.

13. Joubert M, Fourmy C, Henri P, Ficheux M, Lobbedez T, Reznik Y. Effectiveness of continuous glucose monitoring in dialysis patients with diabetes: the DIALYDIAB pilot study. Diabetes Res Clin Pract. 2015;107(3):348-54.

14. Seaquist ER, Anderson J, Childs B, Cryer P, Dagogo-Jack S, Fish L, et al Hypoglycemia and diabetes: a report of a workgroup of the American Diabetes Association and the Endocrine Society. I Clin Endocrinol Metab. 2013;98(5):1845-59.

15. Medtronic MiniMed. Guía del usuario ipro2. Northridge California: Medtronic MiniMed; 2010.p. 117

16. Fresenius Medical Care. Online Clearance Monitoring Assuring the Desired Dose of Dialysis,. Germany: Fresenius medical care; 2007. p. 28.

17. Fresenius Medical Care. Glosario de productos y servicios Mexico [cited 2017 15 de Febrero]. Available from: http://www.fmc-ag.com.mx/glosario.html.

18. Stevens PE, Levin A, Members KDIGOCKDGDWG. Evaluation and management of chronic kidney disease: synopsis of the kidney disease: improving global outcomes 2012 clinical practice guideline. Ann Intern Med. 2013;158(11):825-30

19. Chantrel F, Sissoko H, Képénékian L, Smagala A, Meyer L, Imhoff O, et al. Influence of dialysis on the glucose profile in patients with diabetes: usefulness of continuous glucose monitoring. Horm Metab Res. 2014:46(11):810-3.

20. Meglasson MD. Patent Number: 5,132,324. Use of 3-guanidinopropionic acid in the treatment of non-insulin dependent diabetes mellitus (niddm) United States1992.

21. Eloot $\mathrm{S}$, van Biesen W, Dhondt A, de Smet R, Marescau B, De Deyn PP, et al. Impact of increasing haemodialysis frequency versus haemodialysis duration on removal of urea and guanidino compounds: a kinetic analysis. Nephro Dial Transplant. 2009;24(7):2225-32. 